\title{
RANGO GEOGRÁFICO Y ESTRUCTURA ESPACIAL DE LINAJES GENÉTICOS EN SOPHORA LINEARIFOLIA (FABACEAE), UN ARBUSTO ENDÉMICO DE LAS SIERRAs CENTRALES de Argentina
}

\author{
DAVID ALERCIA ${ }^{1}$, MELISA A. GIORGIS ${ }^{1}$, GUILLERMO FUNES ${ }^{1}$ y ANDREA COSACOV ${ }^{1}$
}

\begin{abstract}
Summary: Geographical range and spatial structure of genetic lineages in Sophora linearifolia (Fabaceae), an endemic shrub of Central Argentina. A large number of endemic species occur in the Sierras of Córdoba and San Luis. A significant group of them shows a disjunct geographic distribution between these two mountain regions. Until the present study, there were none genetic studies that allow us to infer historical processes underlying this geographical pattern. For this purpose, we characterize the distribution area and phylogeographic patterns of Sophora linearifolia, across its geographic range which includes Sierras Chicas (SC) and Cumbres de Gaspar (CG) in Córdoba and in the southwestern area of Sierras of San Luis (SL). We compared the current with the predicted species area of distribution using ecological niche modelling analysis. We also analyzed genetic variability and spatial structure of the DNA chloroplast marker trnH-psbA, and reconstructed the genealogical relationships among the retrieved haplotypes. Current and the predicted distribution agreed in showing a climatic/topographic disjunction between Sierras of Cordoba and San Luis hills, although the current distribution presents a disjunction between SC and CG in Córdoba, not shown in the potential distribution map. We obtained eight haplotypes with restricted distribution and low levels of genetic differentiation that were grouped into four phylogroups: SC, CG and two in SL. Our results indicate that S. linearifolia presents low dispersal ability and it would have recently diversified, suggesting a neoendemic taxon.
\end{abstract}

Key words: Chloroplastidial DNA, biogeography, disjunct, distribution, ecological niche modelling, phylogeography, neoendemic, Sierras Pampeanas.

\begin{abstract}
Resumen: Las sierras de Córdoba y San Luis albergan una gran riqueza de especies endémicas. Un grupo importante de ellas muestra una distribución disyunta entre ambas serranías. Hasta la actualidad no existían estudios genéticos que permitieran inferir procesos históricos asociados a este patrón. Con este propósito caracterizamos el área de distribución y el patrón filogeográfico de Sophora linearifolia, abarcando todo su rango geográfico que incluye las Sierras Chicas (SC) y las Cumbres de Gaspar (CG) en Córdoba y el suroeste de las Sierras de San Luis (SL). Contrastamos la distribución actual con la distribución predicha a partir del modelado del nicho ecológico. Analizamos el nivel de variabilidad genética, la estructuración espacial del marcador de ADN plastidial trnH-psbA y reconstruimos las relaciones genealógicas entre los haplotipos. La distribución actual y la predicha mostraron una disyunción entre Córdoba y San Luis, pero la distribución actual presentó una disyunción entre SC y CG que no fue modelada en la distribución potencial. Se obtuvieron 8 haplotipos de distribución restringida con bajos niveles de diferenciación genética agrupados en 4 filogrupos: SC, CG y dos en SL. Nuestros resultados indican que $S$. linearifolia presentaría baja capacidad de dispersión y que se habría diversificado recientemente, sugiriendo que se trataría de un neoendemismo.
\end{abstract}

Palabras clave: ADN cloroplastidial, biogeografía, distribución disyunta, filogeografía, modelado de nicho ecológico, neoendemismo, Sierras Pampeanas.

1.Instituto Multidisciplinario de Biología Vegetal (IMBIV), CONICET y Facultad de Ciencias Exactas, Físicas y Naturales, Universidad Nacional de Córdoba, Ciudad Universitaria, 5000-Córdoba, Argentina. E-mail: andreacosacov@gmail.com 


\section{INTRODUCCIÓN}

Las sierras de Córdoba y San Luis (en adelante "sierras") representan el extremo austral de las Sierras Pampeanas y se extienden en dirección norte - sur al oeste de la provincia de Córdoba y al noreste de San Luis, entre los $29^{\circ} 30^{\prime}$ y $33^{\circ} 30^{\prime}$ $\mathrm{S}$ y $63^{\circ} 40^{\prime}$ y $65^{\circ} 20^{\prime} \mathrm{O}$. Pueden ser consideradas "islas biogeográficas" dado que ambos cordones serranos están rodeados por tierras más bajas. Se han citado especies endémicas para estas sierras dentro de una vasta variedad de taxones (ej. aves: Nores \& Yzurieta, 1983; anfibios: Lescano et al., 2015; reptiles: Martori \& Aun, 1994; angiospermas: Vischi et al., 2004; Giorgis et al., 2011, Grossi et al., 2011, entre otros). En una reciente contribución al estudio de las angiospermas endémicas, Chiapella \& Demaio (2015) identificaron 89 taxones de angiospermas endémicos de las sierras (69 específicos y 20 infraespecíficos). Entre éstos, aproximadamente el 33\% tienen una distribución disyunta entre las sierras de Córdoba y San Luis; estando mayormente presentes por encima de los $500 \mathrm{msnm}$ en ambas provincias. Esta recurrencia del patrón de endemismos disyuntos en las sierras es indicativa de procesos pasados de alcance regional que afectaron los patrones espaciales de la biodiversidad, en particular para los endemismos de la zona (Lomolino et al. 2006).

Los patrones de distribución endémicos pueden definirse a varias escalas espaciales y taxonómicas en función de los procesos históricos que los han originado. Stebbins \& Major (1965) han definido dos categorías de endemismos: paleoendemismos y neoendemismos. Los primeros corresponden a taxones cuya distribución actual es un vestigio de una distribución mayor en el pasado y están asociados a procesos de retracción de rango. La categoría de neoendemismo es aplicable a especies que han divergido recientemente y está asociada a procesos de expansión del rango geográfico de la misma. En este contexto conocer los tipos de endemismos que se encuentran en una región y reconstruir la historia biogeográfica de los taxones implicados resulta clave para entender los procesos históricos y ecológicos que han modelado su distribución (Lomolino et al., 2006).

Sophora L. (Fabaceae, subfamilia Papilonoideae, tribu Sophoreae) es un género cosmopolita que contiene cerca de 60 especies entre las que se cuentan árboles, arbustos y raramente hierbas perennes, distribuidas en las regiones templadas de casi todo el mundo (Peña \& Cassels, 1996). Sophora cuenta con dos especies citadas para Argentina, ambas endémicas de las Sierras Pampeanas con un acotado rango de distribución: S. rhyncocarpa Griseb. habita los valles húmedos de Salta y Tucumán y $S$. linearifolia Griseb., crece en las Sierras Pampenas del centro de la Argentina, con una distribución disyunta entre las sierras de Córdoba y las de San Luis (Zuloaga et al.,2008). Sophora linearifolia es una especie poco conocida y la literatura disponible sobre ella es insuficiente en muchos aspectos. En cuanto a su distribución geográfica se la ha citado entre los 1400 y $1450 \mathrm{msnm}$ (Ciarlante, 1997) y entre los 1000 y $1500 \mathrm{msnm}$ (Zuloaga et al., 2008). Concerniente a la biología reproductiva de S. linearifolia, se han reportado deficiencias en la microsporogénesis que conllevan a una merma en la producción de semillas viables (Rosenfeldt et al., 2011), aunque la relevancia ecológica de este hecho y su impacto en la capacidad reproductiva de la especie no están claras.

El análisis de los patrones de distribución geográfica de los linajes genéticos de una especie (i.e. filogeografía) es clave para dilucidar los procesos que subyacen a los patrones genealógicos observados, tal como lo demuestran numerosos estudios de este tipo realizados en otras áreas fitogeográficas (ej. Cosacov et al., 2010; Cosacov et al., 2013; Sérsic et al., 2011; Baranzelli et al., 2014). Sin embargo, hasta el momento no existe ningún estudio que utilice esta aproximación en especies endémicas del extremo sur de las Sierras Pampeanas.

En este trabajo nos proponemos caracterizar el rango de distribución geográfica y la estructura espacial de la variabilidad genética a nivel de un marcador del ADN cloroplastidial en S. linearifolia.

\section{Materiales y Métodos}

\section{Especie en estudio y distribución geográfica}

Sophora linearifolia es un arbusto rizomatoso de 0,5 - 1,5 $\mathrm{m}$ de altura endémico de los sistemas serranos de Córdoba y San Luis. Las hojas son seríceo-plateadas, de 5 a $9 \mathrm{~cm}$ con folíolos lineales de $1 \mathrm{~mm}$ o menos de ancho y 15-40 mm de largo. Presenta flores papilionadas, amarillas, de 20 a 


\section{Alercia et al. - Rango geográfico y linajes en Sophora linearifolia}

$25 \mathrm{~mm}$ agrupadas en racimos terminales. El fruto es una legumbre estipitada, subdehiscente uni o pauciseminada (Ciarlante, 1997). A partir de ejemplares herborizados, la base de datos global GBIF (GBIF, 2015) y aportes de los Dres. M. Giorgis, G. Funes, G. Bernardello y M. Tourn (com. pers.) obtuvimos la localización de los sitios con poblaciones de $S$. linearifolia en las provincias de Córdoba y San Luis. Se visitaron todas las localidades registradas y conocidas para la especie de acuerdo a recientes relevamientos florísticos en las sierras de Córdoba (Giorgis et al., 2011; Cantero et al., 2011). Desafortunadamente, no se dispone de información florística comparable para la provincia de San Luis (Cantero com. pers).

\section{Recolección del material}

Coleccionamos material foliar proveniente de individuos de siete sitios en Córdoba (identificados como CO1-CO7) y seis en San Luis (SL1-SL6) (Fig. 1, Tabla 1). En cada sitio se realizó un muestreo sistemático de 5-15 individuos con una separación mínima de aproximadamente $5 \mathrm{~m}$ con el objeto de reducir la probabilidad de muestrear clones. El material vegetal se coleccionó en bolsas herméticas con gel de sílice y se almacenó deshidratado con su correspondiente etiquetado hasta su utilización.

\section{Rango de distribución actual y potencial de la especie}

Dado que la información disponible sobre la distribución de $S$. linearifolia es considerablemente escasa, realizamos un modelado de nicho ecológico (MNE) para aproximar el rango geográfico total y de esta manera evaluar si la distribución potencial inferida a partir de datos climáticos y topográficos también muestra un patrón de distribución disyunto de la especie focal.

Los MNE modelan el nicho fundamental de la especie en función de las características ambientales de los sitios donde la misma ha sido encontrada y luego para cada celda del ráster estima una probabilidad de ocurrencia según la similitud ambiental de esa celda con aquella en donde la especie está presente. Para realizar este análisis se obtuvo la altitud y 19 variables bioclimáticas de la base de datos mundial WorldClim (Hijmans et al., 2005). Con el fin de reducir la colinearidad entre las 20 variables se descartaron las que presentaron una correlación mayor a $|\mathrm{r}|>0,75$ incluyéndose sólo aquellas con un significado ecológico claro (Dormann et al., 2013). Las variables finalmente incluidas en el MNE fueron: temperatura media anual, estacionalidad térmica, temperatura mínima del mes más frío, temperatura media de la estación más fría, estacionalidad de las precipitaciones, precipitación media del trimestre más húmedo y precipitación media del trimestre más seco.

Si bien hay diversos métodos estadísticos para modelar el nicho ecológico, el algoritmo de Máxima Entropía implementado en el programa MaxEnt (Phillips et al., 2006) es uno de los más utilizados y recomendados para este tipo de estudios (e.g., Barry \& Elith, 2006). Utilizamos MaxEnt v.3.3.3k y el análisis se llevó a cabo seleccionando aleatoriamente el $90 \%$ de las ocurrencias para entrenamiento y el $10 \%$ para validación en cada corrida. Se corrieron 10 réplicas con un máximo de 500 iteraciones. El parámetro utilizado para evaluar el valor predictivo del modelo fue el área bajo la curva (AUC, por sus siglas en inglés) que es la probabilidad de que un punto de presencia seleccionado por azar esté ubicado en una celda del ráster con un valor de probabilidad mayor para la ocurrencia de la especie que un punto de ausencia seleccionado al azar. Para graficar la distribución potencial de la especie se utilizó como umbral de corte la menor probabilidad de ocurrencia de un sitio muestreado.

Extracción, amplificación y secuenciación de ADN

Para la extracción de ADN se seleccionaron 4 individuos por sitio muestreado analizándose un total de 52 individuos. El ADN genómico se obtuvo a partir de $0,05 \mathrm{~g}$ de material deshidratado y pulverizado, usando un protocolo CTAB modificado (Doyle, 1987). En cinco individuos representativos de la distribución geográfica de la especie estudiada se comparó la variabilidad de dos regiones no codificantes de cloroplasto rpl32-trnL y trnH-psbA (Shaw et al., 2007) que ya habían sido utilizadas en un análisis filogeográfico previo en otra especie de Sophora por ser los más variables entre numerosos marcadores ensayados (Fan et al., 2013). El espaciador intergénico trnH-psbA (cebadores trnHGUG y psbA) fue el marcador seleccionado para los análisis. La amplificación de las secuencias se condujo bajo las siguientes condiciones: 3 minutos a $94^{\circ} \mathrm{C}$, seguidos de 30 ciclos de 1 minuto a $94^{\circ} \mathrm{C}, 60$ 


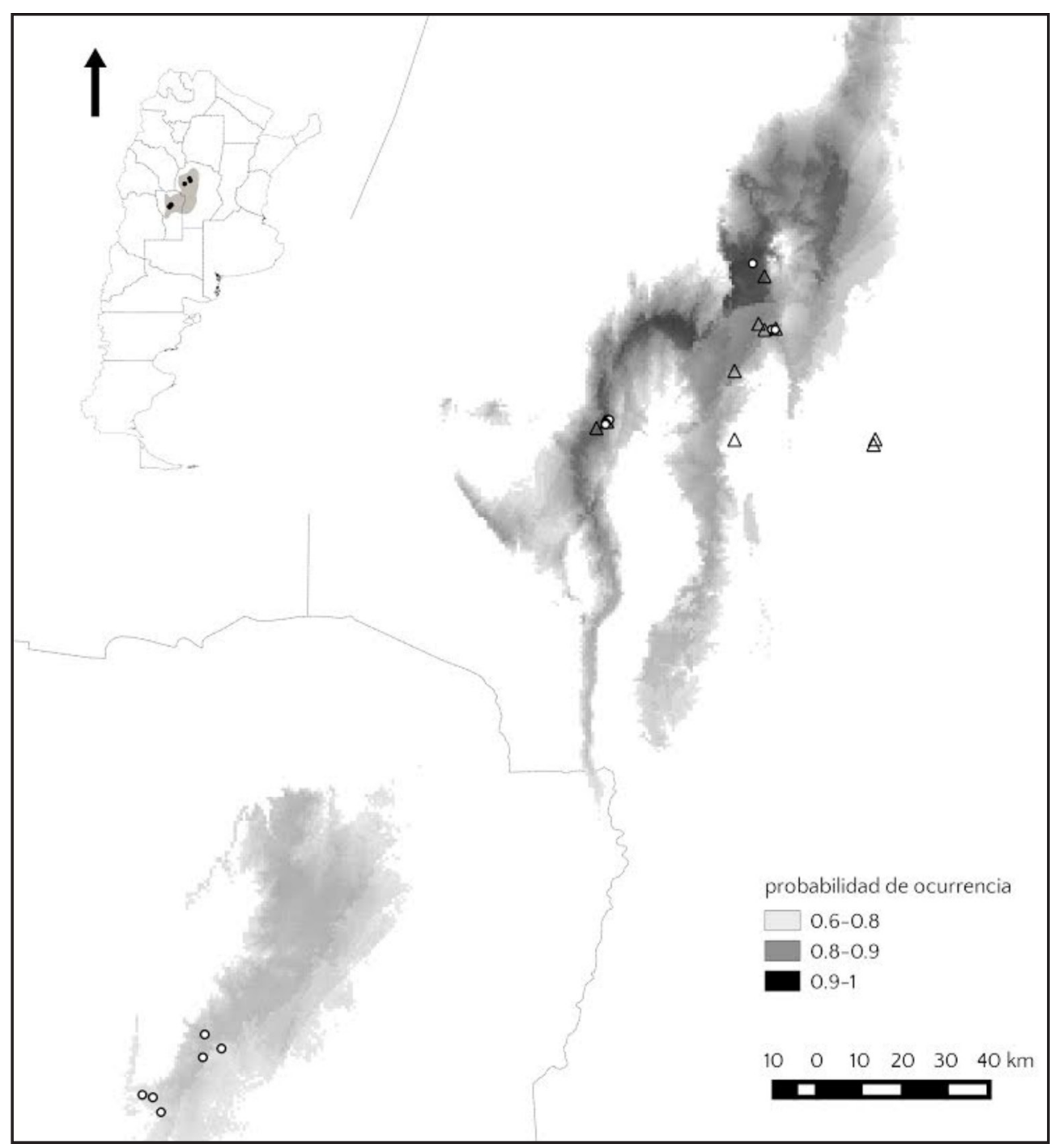

Fig. 1. Mapa del área de estudio mostrando los sitios muestreados (círculos), los registros de herbario (triángulos) y el área de distribución potencial de S. linearifolia, obtenida a partir del modelado de nicho ecológico (MNE). Las áreas sombreadas corresponden a localidades a las que el MNE les asigna una probabilidad de ocurrencia por encima del umbral de presencia utilizado $(P=0,6)$, siendo las áreas más oscuras las de mayor probabilidad.

segundos a $52^{\circ} \mathrm{C}$ y finalmente, un minuto a $72^{\circ} \mathrm{C}$. La eficacia de la PCR fue verificada mediante electroforesis en gel de agarosa al $1 \%$ y los fragmentos amplificados se enviaron a secuenciar con ambos cebadores ("forward" y "reverse") al servicio de secuenciación ofrecido por Macrogen Inc. Las secuencias fueron editadas y alineadas usando los programas ChromasPro v. 1.7.7 (visualización y edición) y BioEdit v. 7.2.5 (Hall, 1999). Cada sitio variable fue verificado visualmente para minimizar errores en el alineamiento. Las inserciones/deleciones de la matriz alineada fueron codificados siguiendo a Simmons \& Ochoterena (2000). 


\section{Alercia et al. - Rango geográfico y linajes en Sophora linearifolia}

Genealogía y distribución geográfica de los haplotipos

Los haplotipos presentes en las 52 secuencias fueron determinados utilizando el Programa DnaSP v.5 (Rozas et al., 2003). Las relaciones genealógicas entre los haplotipos se obtuvieron utilizando el algoritmo Median-Joining implementado en el programa Network v. 4.6. La distribución de los haplotipos por sitios se superpuso en un mapa de la región con el programa QGIS v. 2.8. (QGIS Development Team, 2015).

\section{Estructura poblacional y diversidad genética}

Para inferir la presencia de barreras entre los sitios realizamos un análisis espacial de la varianza molecular -SAMOVA- (Dupanloup et al., 2002). Este análisis considera la información geográfica y genética y genera $\mathrm{k}$ grupos de modo tal de magnificar la componente intergrupal de la varianza y minimizar la componente intragrupo. El análisis se realizó para $\mathrm{k}=2$ a $\mathrm{k}=5$.

Los niveles de polimorfismo para cada sitio y para cada grupo derivado del SAMOVA fueron estimados con los siguientes índices de diversidad molecular: número de haplotipos $(\mathrm{H})$, diversidad nucleótidica $(\pi, \mathrm{Nei}, 1987)$, diversidad haplotídica (Hd, Nei, 1987), promedio de diferencias pareadas (k), y número de sitios polimórficos $(\mathrm{S})$.

\section{Resultados}

\section{Distribución actual y potencial de la especie}

Sophora linearifolia se encuentra en la ladera oeste de las Sierras Chicas y de las Cumbres de Gaspar en la Provincia de Córdoba y en la zona suroeste de las Sierras de San Luis (Fig.1). La especie habita sitios ubicados a una altitud promedio de $1152 \pm 202 \mathrm{msnm}$ con una precipitación media anual de $633 \pm 47,6 \mathrm{~mm}$ y con una temperatura anual media de $14,7 \pm 1,12{ }^{\circ} \mathrm{C}$. La distribución potencial obtenida con el MNE se circunscribe a la región serrana de Córdoba y San Luis y muestra una discontinuidad entre las sierras de Córdoba y San Luis, aunque predice un área continua entre las Cumbres de Gaspar y las Sierras Chicas en la provincia de Córdoba (Fig. 1). El umbral de corte utilizado fue de 0,65 ya que fue la menor probabilidad de ocurrencia asignada a un sitio muestreado.
Genealogía y distribución geográfica de haplotipos

Obtuvimos una matriz alineada de $349 \mathrm{pb}$ con ocho sitios polimórficos. De éstos, cinco corresponden a mutaciones puntuales, dos a inserciones/deleciones de un nucleótido y uno a un fragmento de 19 pb identificado como una inversión. Este último fue eliminado y se codificó como un carácter binario, estando presente en las secuencias de todos los individuos de las localidades SL4, SL5 y SL6 y ausente en el resto. De esta manera ocho sitios polimórficos que se encontraban en esa región fueron reemplazados por un único evento evolutivo. Tal fenómeno ya ha sido reportado para este marcador en otras especies de Angiospermas y se recomienda proceder de la manera en que se trabajó en el presente estudio (Whitlock et al., 2010). Luego de esta edición se identificaron ocho haplotipos entre las 52 secuencias consideradas.

La red de haplotipos muestra que todos los haplotipos se relacionan por un único paso mutacional con su haplotipo genealógicamente más próximo (Fig. 2), siendo la mayor parte de ellos exclusivos de uno o dos sitios geográficamente muy próximos. Se encontraron tres haplotipos en la provincia de Córdoba y cinco en San Luis (Tabla 1). El haplotipo H6, presente en dos sitios (5 individuos) al sur de las sierras de San Luis ocupa una posición central en la red. Los haplotipos de las sierras de Córdoba forman un grupo parafilético con dos linajes derivados del haplotipo H6 -ausente en Córdoba-: H2 y H3 en los sitios de las Sierras Chicas y H1, presente en todos los sitios de las Cumbres de Gaspar. Por su parte, los haplotipos de las Sierras de San Luis conforman un grupo monofilético con dos linajes: H4-H5, que junto a H6 se encuentran al sur de las sierras de San Luis y H7-H8 en los sitios al norte de aquellos. Sólo los sitios SL1 y SL2, localizados al suroeste de las sierras de San Luis, poseen más de un haplotipo por localidad; el resto de las localidades poseen un único haplotipo.

\section{Estructura poblacional y diversidad genética}

El agrupamiento óptimo recuperado con SAMOVA se obtuvo con $\mathrm{k}=4$ (Fig. 3), observándose que la mayor parte de la variación genética $(77 \%)$ es debida a diferencias entre grupos y un $18 \%$ corresponde a diferencias entre sitios dentro de cada grupo (Tabla 2). Los sitios de la provincia de Córdoba conforman dos grupos correspondientes a 


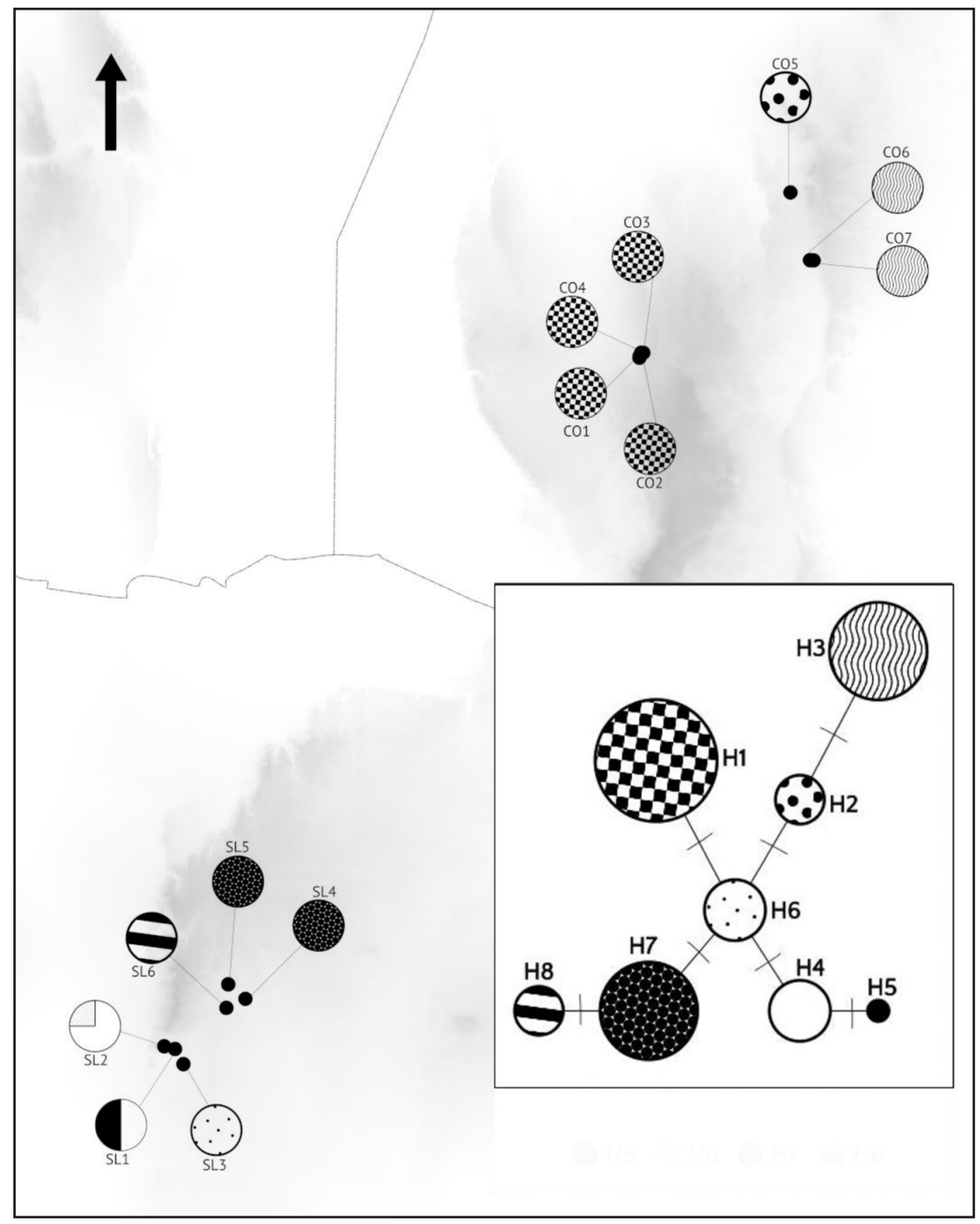

Fig. 2. Distribución geográfica y relaciones genealógicas entre los 8 haplotipos de ADNcl obtenidos a partir del muestreo de 13 poblaciones de Sophora linearifolia. Los gráficos de torta muestran las frecuencias de cada haplotipo en los respectivos sitios, donde la textura de cada haplotipo se corresponde con lo mostrado en el recuadro. En el recuadro se muestra la red de haplotipos construida mediante el algoritmo MedianJoining. Cada haplotipo está representado por un número y un círculo con una textura determinada, cuyo tamaño está en relación a la frecuencia del haplotipo en la muestra total. Cada guión perpendicular a la línea que une a los haplotipos representa un paso mutacional. 


\section{Alercia et al. - Rango geográfico y linajes en Sophora linearifolia}

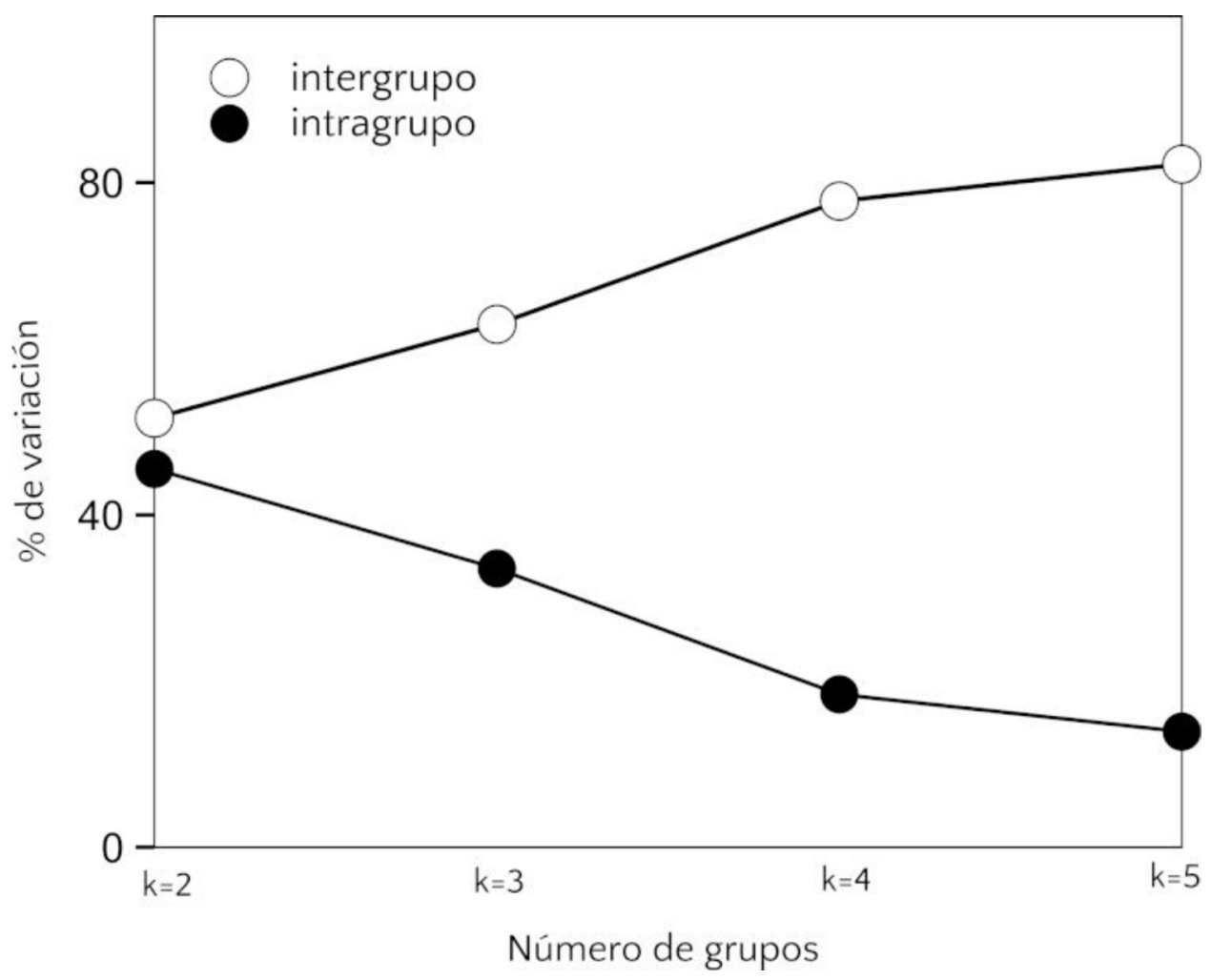

Fig. 3. Resultado del análisis espacial de la varianza molecular. La figura muestra cómo varía el porcentaje de varianza total explicado por cada nivel jerárquico (entre grupos vs. entre sitios dentro de cada grupo) en función del número de grupos genéticos conformados (k). El número óptimo de grupos genéticos se estableció en k=4 ya que maximiza la varianza explicada entre grupos sin que se generen grupos conformados por una sola localidad, lo que sí ocurre a partir de k=5.

las distribuciones en Sierras Chicas y Cumbres de Gaspar. Los sitios de San Luis constituyen también dos grupos: San Luis Sur y San Luis Norte (aquí, "norte y sur" se usan en sentido relativo) (Fig. 2). El grupo con los mayores índices de diversidad genética es San Luis Sur y el menos diverso Cubres de Gaspar (Tabla 3).

\section{Discusión}

Según la información recopilada en este trabajo y en extensos relevamientos florísticos realizados en las Sierras de Córdoba (Cantero et al., 2011; Giorgis et al., 2011) S. linearifolia en la actualidad tiene una distribución restringida a tres áreas: ladera oeste de las Sierras Chicas y Cumbres de Gaspar en la provincia de Córdoba y ladera suroeste de las Sierras de San Luis. El área de distribución potencial de $S$. linearifolia obtenida mediante el modelado de nicho ecológico muestra una clara disyunción entre las sierras de San Luis y las sierras de Córdoba, pero mostró una distribución continua entre las dos áreas ocupadas de la provincia de Córdoba. Un rasgo coincidente de la distribución modelada y la conocida es que ambas se hallan circunscritas al piso altitudinal medio de las sierras (aprox. 1000- $1500 \mathrm{msnm}$ ) rango ya señalado por Zuloaga et al. (2008). Si bien encontramos tres registros de herbario fuera de esta franja, éstos deben tomarse con cautela dada su antigüedad. Dos de ellos corresponden a colecciones de G.H.E.W. Hieronymus de los años 1876 y 1877 localizadas en la ciudad de Córdoba y uno a una colección de A.E. 
Coccuci del año 1961 citada en las cercanías de la localidad de Cuesta Blanca, Dpto. Punilla, por lo que sería muy importante explorar esta última localidad a fin de confirmar su presencia. Sin embargo, la correspondencia de los límites altitudinales del área ocupada y potencial y la caída de la probabilidad de ocurrencia fuera de esos límites, sugeriría que el rango geográfico de $S$. linearifolia respondería a algún tipo de condicionamiento ligado a la altitud, como se evidencia en otros sistemas altitudinalmente restringidos (e.g., Chunco et al., 2013). En relación a la distribución potencial inferida para las sierras de Córdoba, si bien se observa una continuidad climática y topográfica entre ambas serranías hacia el extremo norte de las Sierras Grandes, parecería que este cordón serrano puede constituir, en su porción de mayor altitud, una barrera a la dispersión entre las Sierras Chicas y las Cumbres de Gaspar, como ha sido previamente propuesto (Nores 1995). Es importante mencionar, que el área de ocurrencia predicha para la especie hacia el norte de las Sierras Grandes es un área intensamente estudiada (Giorgis et al., 2011) en la que no se ha encontrado a $S$. linearifolia. La ausencia de la especie en áreas donde el modelado de nicho ecológico arroja altas probabilidades de ocurrencia podría deberse a: un defecto de muestreo en esas zonas, aunque éste no parece ser el caso de $S$. linearifolia, a variables no contempladas en este trabajo, tanto bióticas como abióticas, que podrían tener un rol determinante en la distribución espacial de $S$. linearifolia o bien a que la especie está expandiendo su rango, y no ha arribado aún a lugares aptos para su establecimiento (Soberon \& Peterson 2005).

De manera consistente con la discontinuidad espacial observada, el patrón filogeográfico de secuencias no codificantes de ADN plastidial revela una discontinuidad a nivel genético entre las poblaciones situadas en Córdoba y San Luis. Además, el análisis de estructuración espacial SAMOVA muestra cuatro grupos, dos situados en las sierras de San Luis y dos en las sierras de Córdoba (Sierras Chicas y Cumbres de Gaspar). En términos generales el patrón filogeográfico de la especie se corresponde al modelo III de Avise (2000) caracterizado por presentar haplotipos restringidos geográficamente (i.e. discontinuidad geográfica) sin grandes divergencias genéticas entre ellos (los haplotipos difieren en 1-4 pasos mutacionales). La baja diversidad de haplotipos, y la escasa acumulación de diferencias entre los haplotipos del locus psbA-trnH podría resultar indicativa de

Tabla 1. Sitios muestreados. N: número de muestras, h: número de haplotipos encontrados. ID: identificador del haplotipo (frecuencia absoluta del haplotipo entre paréntesis).

\begin{tabular}{|c|c|c|c|c|c|c|c|}
\hline Provincia & Sitio & Latitud & Longitud & $\begin{array}{c}\text { Altitud } \\
\text { (msnm) }\end{array}$ & $\mathbf{N}$ & h & ID \\
\hline \multirow{7}{*}{ Córdoba } & $\mathrm{CO} 1$ & $-31,3343$ & $-64,94$ & 1464 & 4 & 1 & $\mathrm{H} 1(4)$ \\
\hline & $\mathrm{CO} 2$ & $-31,3346$ & $-64,9349$ & 1464 & 4 & 1 & $\mathrm{H} 1(4)$ \\
\hline & $\mathrm{CO} 3$ & $-31,3434$ & $-64,94475$ & 1441 & 4 & 1 & $\mathrm{H} 1(4)$ \\
\hline & $\mathrm{CO} 4$ & $-31,348$ & $-64,9453$ & 1441 & 4 & 1 & $\mathrm{H} 1(4)$ \\
\hline & $\mathrm{CO5}$ & $-30,8976$ & $-64,5336$ & 1012 & 4 & 1 & $\mathrm{H} 2(4)$ \\
\hline & $\mathrm{CO} 6$ & $-31,0821$ & $-64,4802$ & 1018 & 4 & 1 & $\mathrm{H} 3(4)$ \\
\hline & $\mathrm{CO} 7$ & $-31,0827$ & $-64,4707$ & 1064 & 4 & 1 & H3 (4) \\
\hline \multirow{6}{*}{ San Luis } & SL1 & $-33,2318$ & $-66,2113$ & 1012 & 4 & 2 & $\mathrm{H} 4$ (2) $\mathrm{H} 5(2)$ \\
\hline & SL2 & $-33,2243$ & $-66,2409$ & 971 & 4 & 2 & $\mathrm{H} 4$ (3) $\mathrm{H} 6$ (1) \\
\hline & SL3 & $-33,2731$ & $-66,1886$ & 916 & 4 & 1 & H6 (4) \\
\hline & SL4 & $-33,0951$ & $-66,01955$ & 1026 & 4 & 1 & H7 (4) \\
\hline & SL5 & $-33,0556$ & $-66,0663$ & 1127 & 4 & 1 & H7 (4) \\
\hline & SL6 & $-33,1197$ & $-66,0714$ & 1077 & 4 & 1 & H8 (4) \\
\hline
\end{tabular}




\section{Alercia et al. - Rango geográfico y linajes en Sophora linearifolia}

Tabla 2. Resultados del análisis espacial de la varianza molecular para S. linearifolia con k=4 basado en secuencias de ADNcp; gl: grados de libertad; SC: suma de desvíos al cuadrado; CV: componentes de la varianza; \%V: porcentaje de variación explicada; IF: índices de fijación. $p<0,05$.

\begin{tabular}{|cccccc|}
\hline $\begin{array}{c}\text { Fuente de } \\
\text { variación }\end{array}$ & gl & SC & CV & $\%$ V & IF \\
\hline Intergrupo & 3 & 38,064 & 0,91 & 77,77 & FCT $=0,78$ * \\
Interpoblacional & 9 & 8,167 & 0,21 & 18,4 & $\mathrm{FSC}=0,83$ * \\
\hline Intrapoblacional & 39 & 1,75 & 0,04 & 3,83 & $\mathrm{FST}=0,96$ * \\
\hline
\end{tabular}

procesos de divergencia recientes, facilitados por un bajo flujo genético (en relación al tamaño poblacional efectivo) que habría permitido la diferenciación de haplotipos por efecto de la deriva, tal como ha sido sugerido en otros estudios (e.g., Cosacov et al., 2010).

La ineficacia de la reproducción sexual reportada para $S$. linearifolia y la presencia de rizomas sugieren una estrategia que combina reproducción sexual y vegetativa. Si bien una estrategia similar ha sido observada en otras especies americanas de Sophora (Crowder, 1982) aspectos como la importancia relativa de cada uno de estos mecanismos en la expansión de la especie y las implicancias ecológicas de tal estrategia permanecen como aspectos a dilucidar. Además, la morfología del fruto y el tamaño relativamente grande de sus semillas sugieren una baja capacidad de dispersión (Leishman et al., 2000). Si bien los rasgos señalados respaldan la idea de un bajo flujo genético entre los haplogrupos encontrados queda por determinar si este patrón de variación genética es el reflejo de un muestreo insuficiente, si es idiosincrático del marcador utilizado o, si por el contrario, se verifica también en otros marcadores con una muestra mayor, por lo que la inclusión de otros marcadores al análisis y la intensificación del muestreo resultan aspectos cruciales para esclarecer este aspecto.

Los haplotipos de las sierras de Córdoba conforman un grupo parafilético, ya que el ancestro común de ambos linajes es el haplotipo H6, presente sólo en San Luis, mientras que los linajes de San Luis aparecen conformando un grupo monofilético. Los escenarios biogeográficos que pueden plantearse en este contexto son dos: que los dos filogrupos de las Sierras de Córdoba sean el resultado de dos eventos de colonización independiente del haplotipo H6 y su posterior diversificación o alternativamente, que los filogrupos de Córdoba sean el resultado de un único evento de colonización del H6 y diversificación subsiguiente. En ambos casos, la ausencia del haplotipo H6 en las Sierras de Córdoba podría deberse a un defecto de muestreo o ser el resultado de uno (o más) eventos de extinción. Si bien resulta más probable que ambas poblaciones de la provincia de Córdoba sean el resultado de un único evento, ninguna alternativa puede ser descartada con la información disponible hasta el momento. Nuevos estudios que actualmente están en desarrollo servirán para incorporar información

Tabla 3. Índices de diversidad obtenidos a nivel de especie y para los 4 grupos identificados por

el SAMOVA. N: número de muestras totales; H: número de haplotipos; ID: identidad del haplotipo

(frecuencia relativa entre paréntesis); $\pi$ : diversidad nucleotídica; Hd: diversidad haplotídica; k: promedio de diferencias pareadas; $\mathrm{S}$ : número de sitios polimórficos.

\begin{tabular}{|c|c|c|c|c|c|c|c|c|}
\hline $\begin{array}{c}\text { Grupo } \\
\text { S. linearifolia }\end{array}$ & $\begin{array}{l}N \\
48\end{array}$ & Sitios & $\begin{array}{l}\mathrm{H} \\
8\end{array}$ & ID & $\begin{array}{c}\pi \\
0,005\end{array}$ & $\begin{array}{l}\mathrm{Hd} \\
0,86\end{array}$ & $\begin{array}{c}\mathrm{K} \\
1,92\end{array}$ & $\begin{array}{l}\text { S } \\
7\end{array}$ \\
\hline Cumbres de Gaspar (CG) & 12 & $\mathrm{CO} 2, \mathrm{CO} 3, \mathrm{CO} 4$ & 1 & $\mathrm{H} 1(1,00)$ & 0 & 0 & 0 & 0 \\
\hline Sierras Chicas (SC) & 12 & $\mathrm{CO} 5, \mathrm{CO} 6, \mathrm{CO} 7$ & 2 & $\begin{array}{l}\mathrm{H} 2(0,33) \\
\mathrm{H} 3(0,66)\end{array}$ & 0,001 & 0,48 & 0,48 & 1 \\
\hline San Luis sur (SLS) & 12 & SL1,SL2,SL3 & 3 & $\begin{array}{l}\mathrm{H} 4(0,42) \\
\mathrm{H} 5(0,17) \\
\mathrm{H} 6(0,42)\end{array}$ & 0,002 & 0,68 & 0,83 & 2 \\
\hline San Luis norte (SLN) & 12 & SL4, SL5, SL6 & 2 & $\begin{array}{l}\mathrm{H} 7(0,66) \\
\mathrm{H} 8(0,33)\end{array}$ & 0,001 & 0,48 & 0,48 & 1 \\
\hline
\end{tabular}


de otros marcadores moleculares, lo que nos permitiría concluir sobre los posibles escenarios biogeográficos aquí planteados.

Aunque los resultados aquí presentados son preliminares, nos dan algunos indicios de que las poblaciones actuales de $S$. linearifolia habrían experimentado expansiones y divergencias recientes por lo que podría considerarse a la especie como un neoendemismo y que su distribución actual podría estar condicionada por la estrategia reproductiva de la especie. Además observamos que el patrón disyunto de la especie entre las sierras de Córdoba y San Luis estaría enmarcado por la existencia de una barrera climática y/o topográfica aunque la naturaleza de la misma queda pendiente de esclarecimiento. Estudios previos han sugerido que los patrones de distribución de las especies de la zona serrana podrían haber tomado su configuración actual durante el Cuaternario en el que las variaciones climáticas habrían sido determinantes (Kröhling \& Carignano, 2014; Chiapella \& Demaio, 2015), como ha sido reportado en varios estudios (Cioccale, 1999; Silva et al., 2011; Giorgis et al., 2015, entre otros). Los resultados encontrados para $S$. linearifolia sugieren una diversificación reciente de la especie, posiblemente asociada a procesos geológicos y climáticos recientes en la región. Sin embargo, la datación de los principales eventos de divergencia de los linajes de $S$. linearifolia se hace necesaria para esclarecer el rol de ciertos eventos geoclimáticos pasados en el patrón biogeográfico de la especie.

Vastas regiones de América del Sur, entre ellas la provincia fitogeográfica Chaqueña permanecen prácticamente inexploradas desde la perspectiva filogeográfica (Turchetto-Zolet et al., 2013). Este trabajo constituye un primer acercamiento a la dinámica biótica de las Sierras Pampeanas con un enfoque filogeográfico. Nuestros resultados y conclusiones han de tomarse con carácter preliminar en tanto se refieren a una única especie. En este contexto la elaboración de una filogeografía comparada para la región con la inclusión de otras especies endémicas que compartan la distribución disyunta con $S$. linearifolia, nos permitirá concluir si estos patrones son idiosincráticos de la especie considerada o por el contrario son el resultado de procesos que afectaron de manera similar a la biota de la región.

\section{Agradecimientos}

Este trabajo fue financiado por CONICET (PIP 0456 CO) y constituye parte de la tesina de grado realizada por el Biól. David Alercia. Los autores agradecen a dos evaluadores anónimos por las valiosas sugerencias y comentarios realizados y al IMBIV, al Consejo Nacional de Investigaciones Científicas y Técnicas (CONICET), y a la Universidad Nacional de Córdoba, por las facilidades brindadas para llevar a cabo esta investigación. AC, MAG, GF son investigadores del CONICET y docentes de la UNC.

\section{Bibliografía}

AVISE, J. C. 2000. Phylogeography: The History and Formation of Species. Harvard University Press, Cambridge.

BARANZELLI, M. C., L. A. JOHNSON, A. COSACOV \& A. N. SÉRSIC. 2014. Historical and ecological divergence among populations of Monttea chilensis (Plantaginaceae), an endemic endangered shrub bordering the Atacama Desert, Chile. Evol. Ecol. 28: 751-74.

BARRY, S. \& J. ELITH. 2006. Error and uncertainty in habitat models. J. Appl. Ecol. 43: 413-23.

CANTERO, J. J., J. A. SFRAGULlA, C. NÚÑEZ, A. A. BONALUMI, J. MULKO, A. AMUCHASTEGUI, F. CHIARINI, G. E. BARBOZA \& L. ARIZA ESPINAR. 2011. Flora de los afloramientos de mármoles y serpentinitas de las sierras de Córdoba (Argentina). Kurtziana 36: 11-45.

CHIAPELlA, J. O. \& P. H. DEMAIO. 2015. Plant endemism in the sierras of Córdoba and San Luis (Argentina): understanding links between phylogeny and regional biogeographical patterns. PhytoKeys 47: 59-96.

CHUNCO A.J., PHIMMACHAK S., SIVONGXAY N., STUART B.L. 2013. Predicting Environmental Suitability for a Rare and Threatened Species (Lao Newt, Laotriton laoensis) Using Validated Species Distribution Models. PLOS ONE 8: e59853. doi: 10.1371/journal.pone.0059853.

CIARLANTE, C. 1997. Flora fanerogámica argentina: fasciculo 36. Fabaceae, parte 5. Subfam. 3. Papilionoideae, parte 1. Tribu IX: Sophoreae. Proflora Conicet, Córdoba.

CIOCCALE, M. 1999. Climatic fluctuations in the Central Region of Argentina in the last 1000 years. Quatern. Int. 62: 35-47.

COSACOV, A., L. A. JOHNSON, V. PAIARO, A. A. COCUCCI, F. E. CÓRDOBA \& A. N. SÉRSIC. 


\section{Alercia et al. - Rango geográfico y linajes en Sophora linearifolia}

2013. Precipitation rather than temperature influenced the phylogeography of the endemic shrub Anarthrophyllum desideratum in the patagonian steppe. J. Biogeogr. 40: 168-82.

COSACOV, A., A. N. SÉRSIC, V. SOSA, L.A. JOHNSON \& A. A. COCUCCI. 2010. Multiple periglacial refugia in the patagonian steppe and post-glacial colonization of the Andes: the phylogeography of Calceolaria polyrhiza. J. Biogeogr. 37: 1463-77.

CROWDER, C. A. 1982. Systematics and phylogeny of the herbaceous North American and inland Argentine Sophoras (Fabaceae). PhD Thesis. Graduate Faculty, Texas Tech University, Texas.

DORMANN, C. F., J. ELITH, S. BACHER, C. BUCHMANN, G. CARL, G. CARRÉ, J. R. GARCÍA MARQUÉZ, B. GRUBER, B. LAFOURCADE, P. J. LEITÃO, T. MÜNKEMÜLLER, C. McCLEAN, P. E. OSBORNE, B. REINEKING, B. SCHRÖDER, A. K. SKIDMORE, D. ZURELL \&. S. LAUTENBACH. 2013. Collinearity: a review of methods to deal with it and a simulation study evaluating their performance. Ecography 36: 27-46.

DOYLE, J.J. 1987. A rapid DNA isolation procedure for small quantities of fresh leaf tissue. Phytochemical Bull. 19: 11-15.

DUPANLOUP, I., S. SCHNEIDER \& L. EXCOFFIER. 2002. A simulated annealing approach to define the genetic structure of populations. Mol. Ecol. 11: 2571-81.

FAN, D. M., J. P. YUE, Z. L. NIE, Z. M. LI, H. P. COMES \& H. SUN. 2013. Phylogeography of Sophora davidii (Leguminosae) across the 'Tanaka-Kaiyong Line', an important phytogeographic boundary in southwest China. Mol. Ecol. 22: 4270-88.

GBIF. 2015. Global biodiversity information facility. Disponible en: http://www.gbif.org/ [Acceso: 10 Noviembre 2015].

GIORGIS, M. A., A. M. CINGOLANI, F. CHIARINI, J. CHIAPELLA, G. BARBOZA, L. ARIZA ESPINAR, R. MORERO, D. E. GURVICH, P. A. TECCO, R. SUBILIS \& M. CABIDO. 2011. Composición florística del Bosque Chaqueño Serrano de la provincia de Córdoba, Argentina. Kurtziana 36: 9-43.

GIORGIS, M. A., M. L. LOPEZ, D. RIVERO \& A. M. CINGOLANI. 2015. Cambios climáticos en las sierras de Córdoba (Argentina) durante el holoceno. Aportes a las reconstrucciones climáticas a través del análisis de silicofitolitos del sitio arqueológico El Alto 3. Bol. Soc. Argent. Bot. 50: 361-375.

GROSSI, M. A., N. JULIO, C. N. GARDENAL, J. DI RIENZO \& G. FUNES. 2011. Genetic variability in Apurimacia dolichocarpa (Fabaceae), a narrow endemic species of Córdoba Hills, Argentina. Ann. Bot. Fennici 48: 21-28.
HALL, T. A. 1999. BioEdit: a user-friendly biological sequence alignment editor and analysis program for Windows 95/98/NT. Nucleic Acids Symp. Ser. 41: 95-98.

HIJMANS, R. J., S. E. CAMERON, J. L. PARRA, P. G. JONES \& A. JARVIS. 2005. Very high resolution interpolated climate surfaces for global land areas. Int. J. Climatol. 25: 1965-78.

KRÖHLING, D., CARIGNANO, C.A., 2014, La estratigrafía de los depósitos sedimentarios cuaternarios, en Geología y recursos naturales de la provincia de Córdoba: Córdoba, Argentina, Asociación Geológica Argentina, Relatorio del XIX Congreso Geológico Argentino, 673-724.

LEISHMAN, M. R., I. J. WRIGHT, A. T. MOLES \& M. WESTOBY. 2000. The evolutionary ecology of seed size. En: FENNER, M. (ed.), Seeds: the ecology of regeneration in plant communities, pp. 31-51. CABI International, Wallingford.

LESCANO, J. N., J. NORI, E. VERGA, F. ROBINO, A. BONINO, D. MILOCH, N. RÍOS \& G. C. LEYNAUD. 2015. Anfibios de las Sierras Pampeanas centrales de Argentina: diversidad y distribución altitudinal. Cuadernos de Herpetología 29: 103-115.

LOMOLINO, M. V., B. R. RIDDLE \& J. H. BROWN. 2006. Biogeography. 3rd ed. Sinauer Associates Inc, Sunderland, Massachusetts.

MARTORI, R. \& L. AUN. 1994. Análisis comparativo de la composición de tres comunidades de Squamata de la Sierra Grande de Córdoba, Argentina. Cuadernos de Herpetología 8: 97-103.

NEI, M. 1987. Molecular Evolutionary Genetics. Columbia University Press, New York.

NORES, M. 1995. Insular biogeography of birds on mountain-tops in north western Argentina. $J$. Biogeogr. 22: 61-70.

NORES, M. \& D. YZURIETA. 1983. Especiación en las Sierras Pampeanas de Córdoba y San Luis (Argentina), con descripción de siete nuevas subespecies de aves. Hornero 12: 88-102.

PEÑA, R. C., \& B. K. CASSELS. 1996. Phylogenetic relationships among chilean Sophora species. Biochem. Syst. Ecol. 24: 725-33.

PHILLIPS, S. J., R. P. ANDERSON \& R. E. SCHAPIRE. 2006. Maximum entropy modeling of species geographic distributions. Ecol. Model. 190: 23159.

QGIS DEVELOPMENT TEAM. 2015. QGIS Geographic Information System. Open Source Geospatial Foundation Project. Disponible en: http://www.qgis.org/

ROSENFELDT, S, B. G GALATI, M. P SILVA, G. M TOURN, \& A. L. SCOPEL. 2011. Desarrollo del polen en individuos provenientes de tres 
poblaciones de Sophora linearifolia Griseb. Boletín de la Sociedad Argentina de Botánica (suplemento): 33 Jornadas Argentinas de Botánica.

ROZAS, J., J. C. SÁNCHEZ-DELBARRIO, X. MESSEGUER \& R. ROZAS. 2003. "DnaSP, DNA polymorphism analyses by the coalescent and other methods." Bioinformatics 19: 2496-2497.

SÉRSIC, A. N., A. COSACOV, A. A. COCUCCI, L. A. JOHNSON, R. POZNER, L. J. AVILA, J. W. SITES J. R. \& M. MORANDO. 2011. Emerging phylogeographical patterns of plants and terrestrial vertebrates from patagonia. Biol. J. Linn. Soc. 103: 475-94.

SHAW, J., E. B. LICKEY, E. E. SCHILLING \& R. L. SMALL. 2007. Comparison of whole chloroplast genome sequences to choose noncoding regions for phylogenetic studies in angiosperms: the tortoise and the hare III. Am. J. Bot. 94: 275-88.

SILVA, L.C.R., M. A., GIORGIS, A. MADHUR, L. ENRICO, N. PÉREZ-HARGUINDEGUY, V. FALCZUK, L. TIESZEN \& M. CABIDO. 2011. Evidence of shift in $\mathrm{C} 4$ species range in central Argentina during the late Holocene. Plant and Soil 349: 261-27.

SIMMONS, M. P., \& H. OCHOTERENA. 2000. Gaps as characters in sequence-based phylogenetic analyses. Syst. Biol. 49: 369-81.

STEBBINS, G. L., \& J. MAJOR. 1965. Endemism and speciation in the California flora. Ecol. Monogr. 35: $2-35$.
SOBERON, J., \& PETERSON, A. T. 2005. Interpretation of models of fundamental ecological niches and species' distributional areas. Biodivers. Informatics 2: 1-10.

TURCHETTO-ZOLET, A. C., F. PINHEIRO, F. SALGUEIRO, \& C. PALMA-SILVA. 2013. Phylogeographical patterns shed light on evolutionary process in South America. Mol. Ecol. 22: 1193-1213.

VISCHI, N., E. NATALE, \& C. VILLAMIL. 2004. Six endemic plant species from central Argentina: an evaluation of their conservation status. Biodivers. Conserv. 13: 997-1008.

WHITLOCK, B. A., A. M. HALE, \& P. A. GROFF. 2010. Intraspecific inversions pose a challenge for the trnhpsba plant DNA barcode. PLoSONE 5: http://dx.doi. org/10.1371/journal.pone.001153.3.

ZULOAGA, F. O, O. MORRONE, \& M. BELGRANO (eds.), 2008. Catálogo de las plantas vasculares del cono sur (Argentina, sur de Brasil, Chile, Paraguay y Uruguay). Monogr. Syst. Bot. Missouri Bot. Gard. 107. 3 volumenes, $3486 \mathrm{pp}$.

Recibido el 4 de junio de 2016, aceptado el 13 de septiembre de 2016. 